\title{
Pattern Formation and Stability Issues in Coupled Fuzzy Map Lattices
}

\author{
Horia-Nicolai TEODORESCU ${ }^{1,2}$ \\ ${ }^{1}$ Gheorghe Asachi Technical University of Iasi, \\ 11, Carol I, Iasi, Romania, \\ hteodor@etti.tuiasi.ro \\ ${ }^{2}$ Institute of Computer Science of the Romanian Academy, \\ 2, Codrescu, 700481, Iasi, Romania
}

\begin{abstract}
The aim of the paper is to investigate stability issues and mechanisms of pattern formation in coupled map lattices (CMLs) that use fuzzy nodes. The lattices belong to various topologies, including rings with various vicinities, and linear topologies. The use of fuzzy maps instead of deterministic (crisp) maps in coupled map lattices improves the modelling capabilities and enhances the ability to model complex systems, making CMLs particularly useful for applications where we face uncertainty and imprecision. One of the questions we answer relates to the computational requirements for determining that a periodic pattern of period $p$ develops in a CfML.
\end{abstract}

Keywords: nonlinear dynamics, modelling, fuzzy logic, Lyapunov exponent, patterns

\section{Introduction}

Coupled Map Lattices (CMLs), also named Coupled Map Networks (CMNs), represent a well-established tool in modeling diffusion and flow processes. CMLs are currently used to represent models of such various processes as laminar and turbulent flows, heat propagation, biological population dynamics, and economic processes [8, 11-13, 20-22].

Typical CMLs operating in discrete time are represented by systems of discrete recursive equations with variables with at least two indices, characterized by some form of regular relationship between the variables with indices with close values. The regularity is due to the fact that the nodes in the lattice operate according to influences coming from neighboring nodes, where the neighborhoods are similar $[10,12,27]$, as in Figure 1 . The general equation of a node is written as

$x_{i, t}=f\left(\mathbf{x}_{i, t-1}^{h}\right)$,

Where $x_{i, t}$ is the output of the node with the corresponding indices, $f$ stands for the input-output function of the node and the variable $\mathrm{x}^{h}$ is a vector representing the outputs of the nodes in the vicinity of the node $i$, at previous time moment,

$\mathbf{x}_{i t}^{h}=\left(x_{i-h, t}, x_{i-h+1, t}, \cdots, x_{i, t}, \cdots, x_{i+h, t}\right)$.

In Equations (1) and (2), the index $i$ stands for the position of the node in the row. When the rows are finite, $i=1, \ldots, N$. The index $t$ stands for the time moment; one row in the lattice is assigned to each time moment, $t=0, . ., \infty$, or $t=0, . ., M$ for finite evolutions.

The value $2 h+1=c t$. represents the number of cells (nodes) in the vicinity. The vicinity is assumed symmetrical in (2). A set of initial conditions are needed to solve the equations (1) and (2). The initial conditions represent the values of the variables with the second index 0 , $\mathrm{x}_{i, 0}$; these values are assumed known. The number of indices can be extended at will for creating planar and spatial lattices; for example, two indices can be used to denote space, and the third index is used to denote time.

(a)

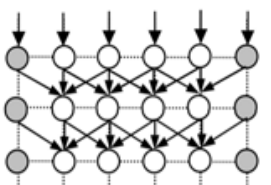

I (b)

Figure 1. (a) Semi-planar coupled map lattice with finite rows; the shaded nodes have boundary condition values. (b) An annular lattice.

The set of equations (1) and (2) can be supplemented with boundary conditions when the lattice is linear and the rows in the lattice have a finite number of nodes. The boundary conditions are either prescribed or reproduce the values of the corresponding boundary nodes on the first row. A CML without boundary conditions is named freely evolving or isolated CML. The boundary conditions refer to constraints imposed to some of the values of the variables, typically to the predetermined values of the "extreme" (boundary) cells, $x_{1 j}=a_{j}, x_{N j}=b_{j}$. 
A simple case of CML uses weighted linear combinations of the outputs of the neighbors, as in multilayer neural networks. Symmetric $w_{i+k}=w_{i-k}$ and antisymmetric $w_{i+k}=-w_{i-k}$ couplings are frequently used [23]. In all the above CMLs, the neighborhood includes elements on both sides, like in Figure 1. This type is named diffusion-type CML. In contrast, if the neighborhood includes only elements on one side, the CML is said to have one-way coupling. These networks are also named unidirectionally coupled map lattices. In case of one-way coupling, a CML with linear nodes has the equation

$$
x_{i}^{j+1}=\gamma \cdot x_{i}^{j}+(1-\gamma) \cdot x_{i-1}^{j} .
$$

Time-delayed map are defined by equations involving time lags larger than 1 , as [23],

$$
x_{i}^{j}=\gamma \cdot f\left(x_{i}^{j-p}\right)+\frac{(1-\gamma)}{2} \cdot\left(f\left(x_{i-1}^{j-p}\right)+f\left(x_{i+1}^{j-p}\right)\right) \text {. }
$$

There are alternative ways of describing CMLs based on graphs. Assume a lattice-type oriented graph. The orientation is interpreted as (time-) propagation. To each node of the graph, attach vicinities (all similar) and a characteristic function. With initial condition for the first line and boundary conditions, we propagate from the first line.

The CML is asymptotically stable when the states of the lines converge when time goes to infinity. When the convergence occurs in a finite number of lines, the behavior is stable. The CML may be periodical, with the states of the lines repeating periodically. CMLS with nonlinear nodes -depending on the initial conditions-may evolve chaotically.

For a process to have a CML model, several conditions have to be fulfilled. In the first place, the process has to have similar components that can be modeled with identical cells, or with a small set of different cells. In the second place, the elements in the process should have a spatial distribution that is regular and corresponds to a simple graph, as a line graph, a circular graph (loop), or a planar regular graph. While more complex graphs have been proposed, computations are easier on simple graphs. Third, the connections between the elements, which model how one element influences the others, should fall in the category of models based on vicinities. Examples of processes that obey these conditions are the atom movements in a crystal, the spins in a magnetic material, the cars on a one-way road, and people sitting in a stadium. To such processes, simple graphs can be attached and a description of the process with a CML is easily done. More examples are introduced in the next section of the paper.

The CMLs described above are deterministic, because the characteristic functions of their nodes were tacitly assumed deterministic ones. CMLs with probabilities are also well-know in physics as models of magnetic and diffusion processes [5, 9, 23].

A generalization of the CMLs, using fuzzy logic systems for describing the behavior of the nodes (cells) in the CML, was introduced in $[25,26]$. The use of fuzzy logic in conjunction with CMLs has several reasons, including the need to simulate networks of human decision makers and the need to deal with dynamic pattern formation and pattern recognition in processes well described by fuzzy logic, as speech [19] and biological signals [30, 31]. There are two cases of CMLs with fuzzy logic cells. In the first, the cells are modeled by FLSs with defuzzification; the second uses nodes modeled by FLSs with fuzzy outputs (no defuzzification at the output).

In Section 2, we present the notion of coupled fuzzy maps and provide the basic equations and examples of applicability. In Section 3, we discuss issues related to pattern formation in crisp and fuzzy CMLs. The last section concludes the paper.

Notations: Throughout this paper, $x_{k}^{t}$ and $x_{k, t}$ denote a variable related to the $k^{\text {th }}$ cell of the row, at time moment $t$. The variable $x$ is used in general for the input and output of cells, the notation $u$ is used for aggregation of input variables, and $f(\cdot)$ denotes the function describing the cell. Whenever the time moment is obvious, we may drop the $t$ index. The symbol $\mu$ is reserved for membership functions (m.f.) and $\beta$ for output singletons in a Sugeno-type FLS. CfML stands for Coupled fuzzy-Map Lattice, fCML stands for fuzzyCoupled Map Lattice, while FLS stands for Fuzzy Logic System. 


\section{CMLs with Fuzzy Logic Cells (CfMLs and fCMLs)}

\subsection{Fuzzy logic nodes}

We recall that a fuzzy logic system is a mapping from a set of m.f.s to another set of m.f.s. FLSs are described by rules, such as "If it heavily rained last nigh, the morning is humid," which include imprecise, qualitative terms, like "heavy rain" and "humid". Reasoning based on such qualitative terms is widespread in human decision-making and everyday life. The m.f.s describe in mathematical terms the "degree of imprecision" and fuzzy logic allows us reasoning based on vague concepts. The reader is referred to textbooks for details.

When the input of the FLS is a number, it is fuzzified and converted into one or several m.f.s. In many cases, fuzzy logic systems are provided with defuzzifiers at the output. The defuzzifier converts a m.f. into a number, in a similar manner a probability distribution function is converted into an estimated value by an estimator (e.g., the average estimator).

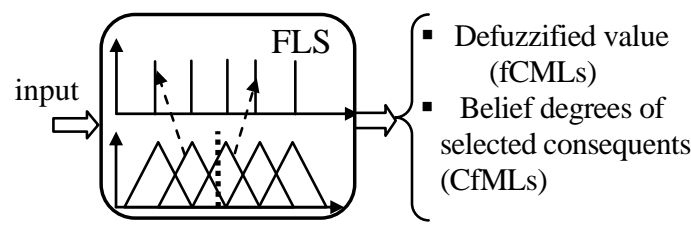

Figure 2. A fuzzy cell in a CfML transfers to its neighbors the defuzzified output value, moreover information on the values of the output m.f.s.

When a FLS is presented with numbers at the input and is provided with defuzzifier, it behaves as a crisp nonlinear system. It can be shown that the nonlinear system has a piecewise input-to-output function. Depending on the definition of the FLS and on the defuzzifier, the characteristic function can be as simple as a linear piecewise function, or a piecewise rational function, or a piecewise transcendental function (for instance, when the input m.f.s are Gaussians).

The so-called Sugeno-type FLS is typically provided with a defuzzifier, see Figure 2 . The difference between fCMLs and CfMLs is illustrated in Figure 2 for nodes represented by Sugeno-systems. In this figure, a Sugeno-type fuzzy system is implementing a node in a CfML. The dotted line shows the position of the input value; the arrows relate the antecedent m.f.s in the rules to the corresponding output singletons. When the FLS is defuzzified, the inputs and outputs of the nodes are real numbers and the resulting CML is a CfML. When the output of the FLS is not defuzzified, both the inputs and the outputs of the nodes are m.f.s, and the lattice is named fuzzy-coupled (fCML). The abbreviation FCML, meaning fuzzy CML, covers both subclasses fCML and CfML.

Before ending this subsection, a few remarks on terms and abbreviations are needed. Rigorously, all lattices using FLSs as nodes should be named coupled fuzzy map lattices, because FLS are fuzzy maps. When the edges of the graph corresponding to the lattice shows the path for a fuzzy variable (m.f.), the corresponding CML should be named fuzzy coupled. Hence, the full name for a fuzzy coupled lattice with nodes represented by FLSs should be fuzzy coupled fuzzy map lattice. To simplify terms and abbreviations, we use the shortcuts fCML and CfML.

\subsection{Applications asking FCML models}

Various illustrations of applying deterministic (non-fuzzy) CMLs to model real-life processes appear in the literature, for instance [24]. Subsequently, we sketch the applicability of FCMLs to real life problems. We rely on the fact that whenever the process involves qualitative reasoning or uncertainty, fuzzy systems are a good toll for modeling the process $[1,7,14,27]$.

Example 1. Consider a line where people are waiting to buy entry tickets to a popular event. They may be discussing various topics. As they stay in line, they can talk and listen only to a few people in their vicinity. This situation corresponds to the line geometry (linear lattice). Successive time moments correspond to successive lines, as in Figure 1 (a). Because natural languages contain imprecise terms and involve uncertainty and qualitative reasoning, the behaviors of human individuals and of social groups are well described by fuzzy logic $[27,29,30,31,32,33]$.

Example 2. Consider a specific trade, like vendors in the railway stations along an isolated railway (with no major stations and rail nodes). They may make decisions about the selling prices for their merchandise based on information they gather about prices at vendors from stations immediately close to their railway station. We deal here with line-type geometry, successive lines - as in Figure 1(a) - 
corresponding to successive time moments. As the decision-making process is often qualitative and based on simple rules [1, 14, 27, 32], it is suitable to apply fuzzy logic to the modeling [1, 14, 27]. Again, we obtain a coupled fuzzy map lattice model. The prices (outputs of the nodes representing the vendors) will be "defuzzified" values of the fuzzy-logic model of the individual vendors (cells in the network); the inputs are the information about prices in, say, the neighboring left and right railway stations. Every cell, standing for a vendor, corresponds to a FLS. Consider that the vendors make the decisions in the form:

If the price of the product at time $t$, in the leftside station, price denoted by $y_{k-1}^{t}$ is somewhat lower $(\mathrm{L})$ than the price she uses, moreover the price of the product in the right-side station, denoted by $y_{k+1}^{t}$, is lower than the price she uses, $y_{k}^{t}$, then increase the own product price $y_{k}^{t+1}$ during the next period of time by $\beta_{1}$. Here, time is assumed discrete and the successive moments of time are denoted by $t, t+1, \ldots$. Notice that somewhat lower has the significance of qualitative appreciation; to this linguistic valuation, we attach a m.f.; moreover, we will use m.f.s for the linguistic degrees somewhat higher, much lower, much higher, and about equal (see Figure 3). The variable of the m.f.s is the difference $d$ between the vendor's and the neighbor's price. Briefly, the above rule is:

If $y_{k-1}^{t}$ is $\mathrm{L}$ and $y_{k+1}^{t}$ is $\mathrm{L}$, then the increment for selling price is $\beta_{1}$, where $\beta_{1}$ is a number.

Similar rules are: If $y_{k-1}^{t}$ is much lower (ML) and $y_{k+1}^{t}$ is $\mathrm{L}$, then the increment is $\beta_{2}$; If $y_{k-1}^{t}$ is higher $(\mathrm{H})$ and $y_{k+1}^{t}$ is $\mathrm{L}$, then increment is $\beta_{3}$.

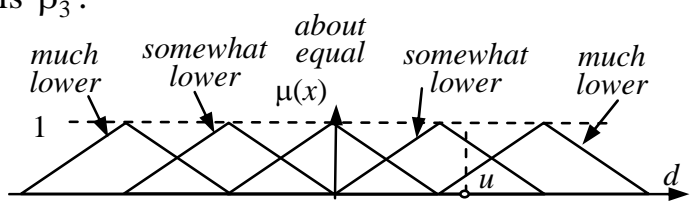

Figure 3. Triangular m.f.s of the fuzzy reference sets. For every input value, $u_{i}$, only (at most) two truth degrees (values of the m.f.s) are nonzero.

The readers can easily add rules to make a complete description of the desired process. The above description corresponds to a Sugeno-type fuzzy system with three inputs.
It is easy to imagine various such descriptions, corresponding to different strategies (market behaviors) of the vendors. For example, a simpler case is that of vendors averaging the prices and deciding as follows:

If the weighted average of the $y_{k-1}^{t}, y_{k}^{t}$, and $y_{k+1}^{t}$ is lower than $y_{k}^{t}$, then the increment of $y_{k}^{t}$ is $\beta_{1}$, where the weighted average is computed as $u^{t}=a_{1} y_{k-1}^{t}+a_{2} y_{k}^{t}+a_{3} y_{k+1}^{t}$.

A description using rules as above is based on weighted averages and corresponds to a singleinput Sugeno fuzzy logic system.

Example 3. As another example, consider a one-way, single-lane road, where all drivers are essentially influenced by the behavior of the drivers in front of them or just behind them - in the three-cell vicinity. The behavior of the drivers manifest in the way they accelerate and decelerate, in the manner they keep the distance with respect to the car ahead of them etc. These "parameters" are imprecisely estimated and perceived by the drivers. For example, imagine that the mood of the drivers is dependent on the manner the nearby drivers behave, moreover that the driving manner depends on the mood. This corresponds to a line-geometry, with cells representing the drivers and behaving according to a quite fuzzy description - a CfML model.

This brief discussion shows that appropriate space distributions of individuals (not restricted to biological individuals), with a limited and uniform vicinity where the influences manifest, may lead to FCML models for processes like decision-making, mood and behavior, and moral opinion. Therefore, developing such tools like FCMLs and analyzing their dynamics may be a useful practice for explaining such processes, predicting their outcome, and correcting (controlling) them. Other similar illustrations of applying FCMLs to modeling are presented in more detail in [25, 26].

\subsection{Basic equations - fCMLs}

The fCMLs make use of the intrinsic properties of the fuzzy cells by carrying fuzzy information (FI) from one cell to the cell on the next row. The type of FI sent to the next row and the way of aggregating this information with other pieces of information define the CfMLs and their peculiarities. 
Under a general framework, in a fuzzy-coupled map lattice the values of the m.f.s of the cells $[25,26]$ are functions of the fuzzy output values of the neighboring fuzzy cells on the previous row, that is, they are functions with variables the values of all the output m.f.s of the neighboring fuzzy cells on the previous row. All nodes are assumed identical and having the same variable for all the input and output m.f.s. The general equation of CfMLs is then:

$$
\begin{array}{r}
\mu_{i, t+1}^{j}(v)=f_{j}\left(\mu_{i-h, t}^{1}(v), \cdots, \mu_{i+h, t}^{1}(v), \cdots,\right. \\
\left.\mu_{i-h, t}^{r}(v), \cdots, \mu_{i+h, t}^{r}(v)\right)
\end{array}
$$

where $j=1, \ldots, r$ is the index of the m.f.s, $\mu_{i, t+1}^{j}(v)$ is the value of the $j^{\text {th }}$ m.f. of the $i^{\text {th }}$ fuzzy cell on the $t^{\text {th }}$ row, $v$ is the variable of the m.f.s, and $f_{j}($.$) is one of the j$ functions defining the cells. Figure 4 illustrates the idea in Equ. (5) for the case of a 3-vicinity and FLSs with 3-m.f. outputs.

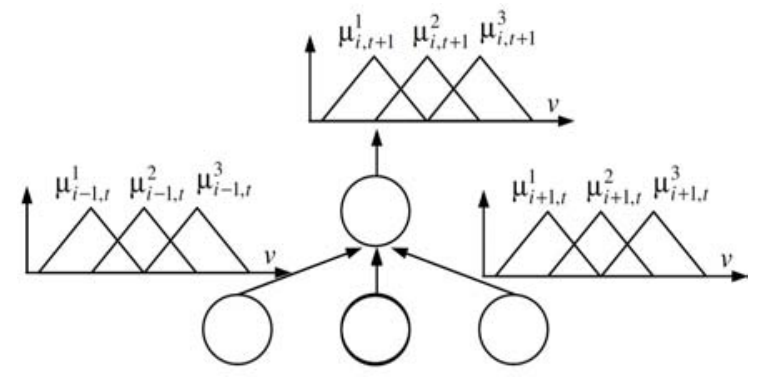

Figure 4. Output triangular m.f.s of the nodes.

Example 1. Consider Mamdani-type fuzzy system nodes with three triangular m.f.s at the output. Define the values of the output m.f.s determined by the ad hoc formula

$$
\begin{aligned}
& \mu_{i, t+1}^{j}(v)= \\
& =\max \left(\max \left(\mu_{i-1, t}^{j}(v), \mu_{i, t}^{j}(v), \mu_{i+1, t}^{j}(v)\right),\right. \\
& \left.\quad \min \left(\mu_{i-1, t}^{r \neq j}(v), \mu_{i, t}^{r \neq j}(v), \mu_{i+1, t}^{r \neq j}(v)\right)\right) .
\end{aligned}
$$

Together with the expressions of the m.f.s, the above relation defines a fCML.

Example 2. Consider a Sugeno system having three Gaussian input m.f.s

$$
\begin{aligned}
& \mu^{j}(x)=\exp \left(-\left(x-a_{j}\right)^{2} / s_{j}\right), \\
& j=1,2,3 ; \quad a_{j}, \quad s_{j} \in \mathbf{R}, \quad s_{j}>0 .
\end{aligned}
$$

The corresponding output singletons are denoted by $\beta^{j}$. A node from the layer $t$ produces three singletons with the corresponding truth degree,

$$
\tilde{\mathbf{y}}_{i, t}=\left\{\left(\beta_{i, t}^{1}, \theta_{i, t}^{1}\right),\left(\beta_{i, t}^{2}, \theta_{i, t}^{2}\right),\left(\beta_{i, t}^{3}, \theta_{i, t}^{3}\right)\right\}
$$

We assume that the three inputs to the node $i$ on the layer $t+1$, each input represented by the fuzzy vector $\tilde{\mathbf{y}}$, are dealt with according to the standard (min-max) fuzzy logic. Then, the aggregation operation produces an input to the node $i$ on the layer $t+1$ according to:

$$
\begin{aligned}
\tilde{\mathbf{x}}_{i, t+1}=\{ & \left(\beta_{i, t}^{1}, \max \left(\theta_{i-1, t}^{1}, \theta_{i, t}^{1}, \theta_{i+1, t}^{1}\right)\right), \\
& \left(\beta_{i, t}^{2}, \max \left(\theta_{i-1, t}^{2}, \theta_{i, t}^{2}, \theta_{i+1, t}^{2}\right)\right), \\
& \left.\left(\beta_{i, t}^{3}, \max \left(\theta_{i-1, t}^{3}, \theta_{i, t}^{3}, \theta_{i+1, t}^{3}\right)\right)\right\} .
\end{aligned}
$$

Because we assumed that all nodes are identical, the singletons should be identical independent of the indices $i, t$. Therefore, we drop these indices. The truth values of the singletons remain dependent on all indices. We can write (8) as

$$
\left.\tilde{\mathbf{x}}_{i, t+1}=\left\{\left(\beta^{1}, \theta_{i, t+1}^{1}\right),\left(\beta^{2}, \theta_{i, t+1}^{2}\right),\left(\beta^{3}, \theta_{i, t+1}^{3}\right)\right)\right\}
$$

where $\left.\theta_{i, t+1}^{j}=\max \left(\theta_{i-1, t}^{j}, \theta_{i, t}^{j}, \theta_{i+1, t}^{j}\right)\right)$.

The node $i$ on the layer $t+1$ responds with the fuzzy output vector of singletons according to (7),

$\tilde{\mathbf{y}}_{i, t+1}=\left\{\left(\beta_{i, t+1}^{1}, \theta_{i, t+1}^{1}\right),\left(\beta_{i, t+1}^{2}, \theta_{i, t+1}^{2}\right),\left(\beta_{i, t+1}^{3}, \theta_{i, t+1}^{3}\right)\right\}$

where, using (6),

$\theta_{i, t+1}^{j}=\max \left(\mu^{j}\left(\beta^{j}\right), \theta_{i, t+1}^{j}\right)$.

The equation (9) completes the computation of the fuzzy node.

\subsection{Basic equations - CfMLs}

Formally, equation (5) holds for CfMLs too. However, the details of application of the equation (5) differ and take into account the type of FLS used for the nodes and the logic used. Considering the case of standard Sugenotype, identical nodes with defuzzified output, for 3-input nodes, as in the previous example, the output is a function of the values of the m.f.s only:

$x_{i, t+1}(u)=\frac{\mu^{1}(u) \beta^{1}+\mu^{2}(u) \beta^{2}+\mu^{3}(u) \beta^{3}}{\mu^{1}(u)+\mu^{2}(u)+\mu^{3}(u)}$.

In (10), $u$ is a value determined by the aggregation of the defuzzified outputs of the 
nodes on the previous line. A typical aggregation is the weighted sum,

$x_{i, t+1}=w_{-1} x_{i-1, t}+w_{0} x_{i, t}+w_{1} x_{i+1, t}$,

where $w_{k}$ are weights.

\section{Pattern Formation in CMLs and Fuzzy CMLs}

The formation of patterns in CMLs is extensively documented in the literature, e.g [2$5,11,12,15-17]$. Stability issues in various types of CMLs have been addressed using various tools in [2-6, 15-17, 21]. Patterns in both fCMLs and CfMLs have been presented in [25, $26,28,32]$. We present subsequently examples of patterns that are obtained similarly to those in [26]; because the FCMLs used are similar to those in [26], we do not discuss them here.

We exemplify the discussion with pictures of the behaviors of the CfMLs, as shown in Figures 5-7. In these figures, the time axis is along the horizontal direction, the first line is the vertical at the left-side of the picture, and the narrow strip at the left of the pictures shows (enlarged) the first line, that is, the initial condition used in the simulation. The initial condition is generated randomly. All pictures are converted from color to gray and have modified contrast for pattern visibility.

Figure 5 exemplifies the formation of stable (solid arrows) and vanishing (thinner, contour arrows) strip-like patterns. Figure 6 shows the development of almost randomly distributed triangular patterns along triangle-formatted strips.

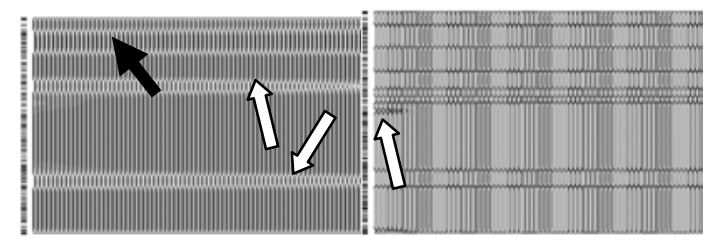

Figure 5. Stable strip-like pattern. Right: Vanishing (light arrow) and stable strips

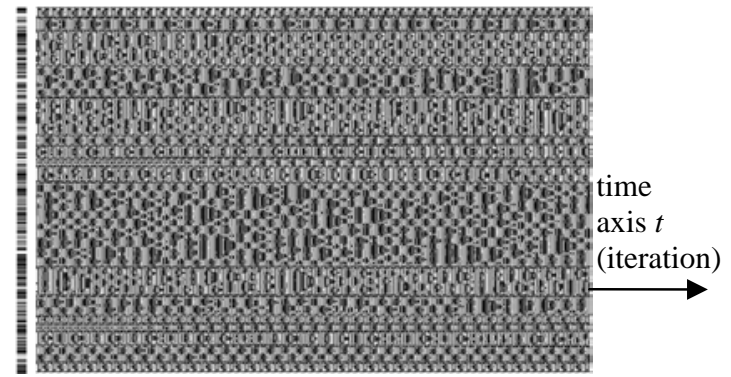

Figure 6. The formation of triangular patterns

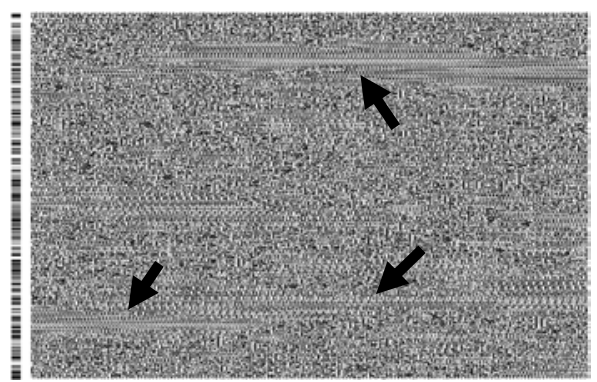

Figure 7. Spatio-temporal coalescence (arrows).

Notice that the triangular patterns represent a form of local (line) self-organization, which is symmetrically "erased" toward its center during time. Finally, Figure 7 illustrates cases of coalescence (partial organization). The remaining part of the paper is devoted to explain these self-organization processes.

Many properties of the CMLs and CfMLs are due to the lattice geometry, not to the cell (node) properties, or to a combination of the two. For example, the formation of the triangular patterns is directly related to the type of vicinity and to the type of the lattice, and partly independent of the function performed by the nodes. Constant strips appear when localized constant solutions are allowed. Periodicity related to the lattice graph and vicinity type helps explaining the periodically varying strips. Determining what patterns the CfMLs can develop is of interest because it helps us to make predictions on the dynamic behavior of the modeled process.

\subsection{Conditions for periodic patterns}

A CML has period $p$ if the output of the node $j$ on line $n$ is the same to the output of the node $i$ on line $n+1, y_{i, n}=y_{i, n+p}\left(\forall_{i}, \forall_{n}\right)$. We assume a 3vicinity. Then, detailing the meaning of the periodicity condition requires recursively developing the expression of $y_{i, n+p}$ as a function of $2 p+1$ values $p$ lines behind, $y_{i-p, n}, \cdots, y_{i, n}, \cdots, y_{i+p, n}$. Indeed,

$$
\begin{aligned}
& y_{i, n+p}=f\left(y_{i-1, i+p-1}, y_{i, n+p-1}, y_{i+1, n+p-1}\right)= \\
& =f\left(f\left(y_{i-2, n+p-2}, y_{i-1, n+p-2}, y_{i, n+p-2}\right),\right. \\
& \left.\quad f\left(y_{i-1, n+p-2}, y_{i, n+p-2}, y_{i+1, n+p-2}\right)\right)=\cdots
\end{aligned}
$$

Notice in Figure 8 that for 2-period, $y_{k, n+2}=y_{k, n}$, five initial values (line $p$ ) contribute to the final value (line $p=2$ ), each initial value going through two edges of processing by a function $f$. When the inputs to nodes are aggregated in an invertible manner, that is, when the three 
input values $y_{k-1, n}, y_{k, n}, y_{k+1, n}$ produce an input value specific only to the vector $\mathbf{y}_{k, n}=\left(y_{k-1, n}, y_{k, n}, y_{k+1, n}\right)$, the periodicity condition along the $k$ index, $y_{k, n+2}=y_{k, n}$, implies the periodicity of the corresponding input vector $\quad \mathbf{y}_{k, n}=\left(y_{k-1, n}, y_{k, n}, y_{k+1, n}\right)$. Consequently, in this specific case of aggregation, 2-periodicity along $k$ implies periodicity of the whole set of 5 values $y_{k-2, n}, \cdots, y_{k+2, n}$. The latter condition is equivalent to a periodicity in a strip of indices $k-2, k-1, k, k+1, k+2$. It is easy to see that propagating along the $k$ indices the same conditions as above, the whole line of index $n$ must be periodical with the same period. This explains the tendency of CMLs and FCMLs to develop line periodicity, as seen in Figure 5. Notice however that the invertible aggregation may occur in fCMLs, but invertibility is not satisfied in typical CMLs and in CfMLs with aggregation performed by weighted sums as in (11).

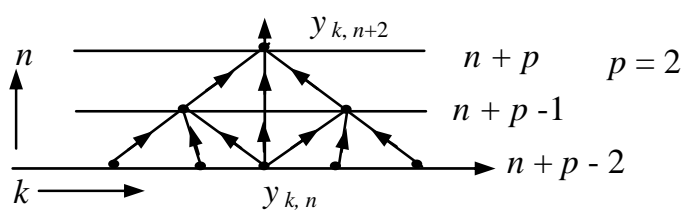

Figure 8. The simplest case of periodicity, 2-period $(p=2)$.

The lower the period, the smaller is the number of conditions in the form of (12) to be satisfied by the CML. This explains why small period patterns appear more frequently in simulations of CMLs. In general, for a $p$-period, the number of conditions to be satisfied in a lattice with 3vicinity and invertible node function is $2 p+1$ for the central node (see Figure 9), moreover a number of $(2 p+1) \times(2 p+1)$ conditions must be satisfied to guarantee that the $2 p+1$ points on the row $n-p$, which determine the periodicity condition for the central node on the row $n$, are periodically evolving.

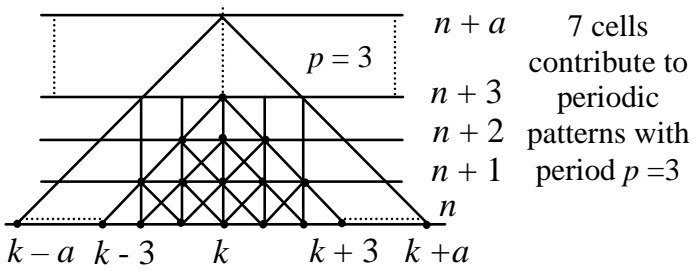

Figure 9. The 3-period case of periodicity, for infinitely large lattices
The above discussion shows that small periods are more probable in CMLs with invertible node functions.

\subsection{Formation of small triangular patterns}

The above discussion also explains the formation of triangular patterns that spontaneously form and die out, as in Figure 6. Indeed, suppose that a number of 7 adjoin nodes on a row of a CML have the same value, moreover that value $y$ satisfies the condition $f(x, x, x)=x$. Then, the value $x$ is guaranteed to survive in the triangle whose basis is composed of the 7 nodes, see Figure 6, but not more than in the triangle. That is, a triangular pattern forms, then dies out. Smaller or larger triangular patterns are produces in a similar way.

The occurrence of 7 nodes with the same value is improbable in a crisp CML. However, the condition of equality can be relaxed to the conditions $y_{k-3, n}, \cdots, y_{k+3, n} \in I$, where $I$ is a (small) interval, and $f\left(y_{s-1, r}, y_{s, r}, y_{s+1, r}\right) \in I$, $\left\{y_{s-1, r}, y_{s, r}, y_{s+1, r}\right\} \subset \subset\left\{y_{k-3, n}, \cdots, y_{k+3, n+}\right\}$, that is, in all relevant vicinities. The above conditions are easily satisfied in fCMLs and CfMLs, because of the operation based on m.f.s, which inherently are selecting intervals.

\subsection{Row symmetry preservation and periodicity issues}

In this subsection we consider CMLs with finite rows. We show that in these CMLs, the effects occurring at the edges of the lattice are essential in determining the lattice behavior. In this context, we address the following questions: i) If and when should we expect that a symmetric row is transformed into a symmetric row; ii) How many rows should we compute to make sure a specific pattern, like periodicity, or strip-like pattern is preserved? iii) Can the answers to the above questions be independent on the cell function $f$ ?

The following properties hold:

Property 1. In a lattice with identical cells, 3vicinity, with finite rows of width $2 N+1$, with no boundary conditions (free boundaries) or with symmetric boundary conditions, a symmetric row necessarily generates a symmetric row. The property is true for crisp CMLs, fCMLs and CfMLs. 
The property results from the conditions imposed and from the symmetry of the vicinity and from the expression of the node function. Because of the symmetry of the row with index $t$, the output of the nodes $y_{N+1-s, t}$ and $y_{N+1+s, t}$ are equal, for all $1 \leq s \leq N$. Therefore, whatever type of aggregation function $g()$ is used by the nodes, the following equality holds,

$$
\begin{aligned}
& y_{N+1-s, t+1}=f\left(g\left(y_{N-s, t}, y_{N+1-s, t}, y_{N+2-s, t}\right)\right)= \\
& =y_{N+1+s, t+1}=f\left(g\left(y_{N+s, t}, y_{N+1+s, t}, y_{N+2+s, t}\right)\right) .
\end{aligned}
$$

As a consequence of the above property, once a symmetric row is created or spontaneously occurs in a CML or FCML, only half of the structure needs to be computed thereafter. Notice that the property holds whatever are the aggregation and node functions.

Property 2. Under the same assumptions as in Property 1 (no boundary conditions case only), if two rows of the lattice, with indices $t$ and $t+4 N+1$ are equal, then the CML has period $4 N+1$. The property does not depend on the type of nodes (crisp or fuzzy) and holds for both fCMLs and CfMLs.

The proof of the property relies on geometrical considerations. A node on the border of the lattice, as node $\mathbf{B}$ in Figure 10, propagates its influence through connections to other nodes until the other border, in node $\mathbf{C}$ placed exactly after $2 N$ lines. On whatever other allowed path we determine the influence of the node $\mathbf{B}$ on a node placed on the line $t+2 N+1$, the path has a length of exactly $2 N$ edges in the lattice, see Figure 10. The sub-lattice delimited by the rows $t$ and $t+2 N+1$ is thus performing as an elementary block of the lattice.

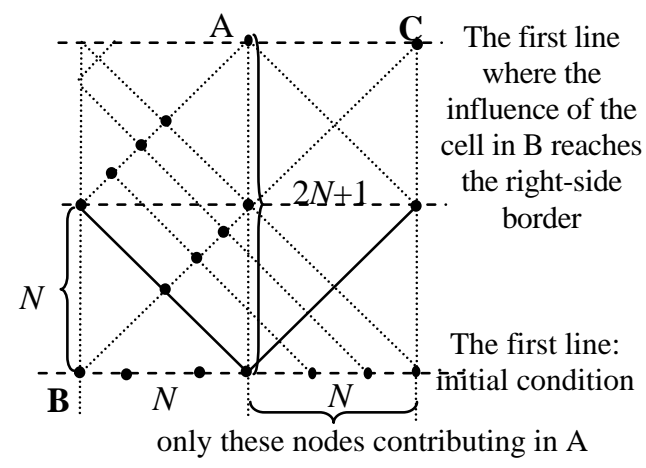

Figure 10. The boundary-reflection and boundaryto-boundary driven pattern formation

Consider two successive block of $2 N+1$ rows, with the last row of the first block identical to the first row of the second block. Then, it is apparent from Figure 10 that after $4 N+1$ rows, the paths from any node on the line $t$ and any other node on the line $t+4 N+1$ repeat. We name the value $4 N+1$ inherent lattice period.

Because of the repetition proved above, whenever two lines with indices $t$ and $t+4 N+1$ are equal, we are guaranteed of a period $4 N+1$ and can stop computations of the lattice. The search of periodicity in a lattice with large $N$ is thus simplified, because instead computing, say $100 \mathrm{~N}$, we need to compute only $4 N+1$ lines if a line repeats. Because the simulation of the CMLs and of the networks of fuzzy systems is computation intensive [18, 28, 32], any result that reduces the computation load, as the above one, helps in the implementation.

Property 3. Under the same assumptions as in Property 1, if two rows of the lattice, with indices $t$ and $t+Q \times(4 N+1)$ are equal, then the CML has period $Q(4 N+1)$.

The property follows from the same considerations as for Property 2.

As a practical consequence of the above properties, no simulation shall include less then $2 N+1$ rows, to derive a viable conclusion on the pattern formation. The second and third properties hold for fCMLs and CfMLs as well. These properties provide an indication on the number of rows to be computed for determining if itinerant chaotic behaviors occur. Because the basic structure of the bounded lattices has $4 N+1$ rows, at least multiple of a few tens of $4 N+1$ lines must be computed.

\section{Discussion and Conclusions}

Following [26], we thoroughly discussed the concepts related to coupled map lattices that use FLSs as nodes of the lattice. Special emphasis was placed on examples of processes that can be modeled with fuzzy coupled map lattices. We have stressed that such lattices are appropriate to model social and economical processes; moreover, we provided simplified, but viable examples for such processes.

The rational of using FLSs as cells in the CMLs is multi-fold. In the first place, FLSs are suitable to model systems with inherent imprecision or with uncertainties. Moreover, FLSs with defuzzifier represent universal 
approximators for smooth functions on bounded intervals. Consequently, there is a guarantee that a large range of applications is treatable with such models.

We provided explanations for several features occurring in crisp and fuzzy CMLs, such as triangular patterns, strips, and periodicity. We determined necessary conditions for periodicity in linear CfMLs and conditions for the minimal number of iterations in simulations of CfMLs to determine if a period $p$ occurs. Estimations of the required number of iterations to determine periodicities in CMLs have been derived. The results related to patterns formation are valid for crisp as well as fuzzy coupled map lattices.

\section{Acknowledgments}

A section of this paper was presented in a preliminary form at the ICCS 2007 Conference, Boston, but remained unpublished. Issues from another subsection were briefly presented in [28]. Research partly supported by the Romanian Academy and by two grants from the Ministry of Education and Research, Romania.

\section{REFERENCES}

1. ADRIAENSSENS, V., B. DE BAETS, P. GOETHALS, N. DE PAUW, Fuzzy RuleBased Models for Decision Support In Ecosystem Management. Science of the Total Environment, Vol. 319, (1-3), 2004, pp. 1-12.

2. AMRITKAR, R. E., J. SARIKA, H., CHIN-KUN, Synchronized Clusters in Coupled Map Networks. II. Stability Analysis Physical Review E, Vol. 72, 1, 016212_1-016212_9, 2005.

3. AMRITKAR, R. E., J. SARIKA, H., CHIN-KUN, Synchronized Clusters in Coupled Map Networks. II. Stability Analysis. Physical Review E Vol. 72, 016212, 016212-1 -- 016212-9, 2005.

4. ATAY, F. M., O. KARABACAK, Stability of Coupled Map Networks with Delays, SIAM J. Appl. Dyn. Syst., Vol. 5, 3, 2006, pp. 508-527.

5. DE AGUIAR, M. A. M., I. R. EPSTEIN, Y. BAR-YAM, Analytically Solvable Model of Probabilistic Network Dynamics, Physical Review E, 772, 067102, 067102-1- 067102-4, 2003
6. DUBOIS, D., H. FARGIER, H. PRADE, P. PERNY, Qualitative Decision Theory: from Savage's Axioms to Nonmonotonic Reasoning. Journal of the ACM (JACM), Vol. 49, 4, 2002, pp. 455-495.

7. ATAY, F. M., J. JOST, A. WENDE, Delays, Connection Topology, and Synchronization of Coupled Chaotic Maps. Phys. Rev. Let., 92, 144101, 2004.

8. GALLUCIO, A., New Algorithm for the Ising Problem: Partition Function for Finite Lattice Graphs, Physical Review Letters, Vol. 84, 26, 2000, pp. 5924-59277.

9. GHEORGHIU-SVIRSCHEVSKI, S., Y. BAR-YAM, Multiscale Analysis of Information Correlations in an InfiniteRange, Ferromagnetic Ising System, Phys. Rev. E, Vol. 70, 6, 066115, 2004

10. JIANG M., Q. ZHANG, A Coupled Map Lattice Model of Tree Migration. Discrete Contin. Dyn. Syst. B, Vol. 9, 1, 2008, pp. 83-101.

11. KADJI, E., J. C. OROU, P. WOAFO, Spatiotemporal Dynamics in a Ring of $\mathrm{N}$ Mutually Coupled Self-Sustained Systems. Chaos, Vol. 17, 033109, 2007

12. KANEKO, K., Simulating Physics with Coupled Map Lattice. Formation, Dynamics and Statistics of Patterns 1, World Scientific, Singapore, 1990.

13. KANEKO, K., Overview of Coupled Map Lattices. Chaos, Vol. 2, 3, 1992., pp. 279-282

14. LEE, C. C., Fuzzy Logic in Control Systems: Fuzzy Logic Controller, Part II, IEEE Trans. Systems, Man, Cybernetics, Vol. 20, 2, 1990, pp. 419-435.

15. LI X., G. CHEN, Synchronization and Desynchronization of Complex Dynamical Networks: An Engineering Viewpoint. IEEE Trans. Circuits and Systems-I: Fundamental Theory and Applications, Vol. 50, 11, 2003, pp. 1381-1390

16. MANRUBIA, S. C., A. S. MIKHAILOV, Mutual Synchronization and Clustering in Randomly Coupled Chaotic Dynamical Networks. Physical Review E, Vol. 60, 1999, pp. 1579-1589. 
17. MANRUBIA, S. C., A. S. MIKHAILOV, Very Long Transients in Globally Coupled Maps. Europhysics Letters, Vol. 50, 2000, pp. 580-586

18. RESTEANU C., R. TRANDAFIR, Programming Problems with a Large Number of Objective Functions. Dimov I, Dimov S, Kolkovska N. (Eds.), Numerical Methods and Appl., Lecture Notes Comp. Sci., Vol. 6046, 2011, pp. 207-214.

19. RODRIGUEZ, W., H. N. TEODORESCU, F. GRIGORAS, A. KANDEL, H. BUNKE, A Fuzzy Information Space Approach to Speech Signal Non-linear Analysis. Int. J. Intelligent Syst. Vol. 15, 4, 2000, pp. 343-363

20. RUDZICK O., PIKOVSKY, A., Unidirectionally Coupled Map Lattice as a Model for Open Flow Systems. Physical Review E Vol. 54, 5, 1996, pp. 5107- 5115.

21. SAYAMA, H., L. KAUFMAN \& BARYAM, Spontaneous Pattern Formation and Diversity in Spatially Structured Evolutionary Ecology, In: Focus on Biodiversity Research, J. Schwartz (Ed.), 1-16, Nova Science Publishers, Inc., 2006

22. SEKI M., K. ITO, A Coupled Map Lattice for Geomagnetic Polarity Reversals That Exhibits Realistic Scaling, Earth Planets Space, Vol. 51, 1999, pp. 395-902.

23. SPROTT, J. C., G. ROWLANDS, Chaos Demonstrations. User's Manual. American Institute of Physics, N.Y., 1995

24. SCHUMANN, A. W., Q.U. ZAMAN, Software Development for Real-Time Ultrasonic Mapping of Tree Canopy Size. Comp. \& Electr. Agric., vol. 47, 2005, pp. 25-40.

25. TEODORESCU H. N., Coupled Map Networks and Uncertainty propagation. Int. J. Chaos Theory \& Appl., Vol. 8, 1, 2002, pp. 3-16.

26. TEODORESCU H. N., Self-organizing Uncertainty-based Networks. 131-160. In: P. Melo-Pinto, H.-N. Teodorescu and T. Fukuda (Eds.), Systematic Organisation of Information in Fuzzy Systems, NATO Sci. Series III, Vol. 184, 2003, pp. 131-159.
27. TEODORESCU H. N., Information, Data, and Information Aggregation in Relation to the User Model. In MeloPinto P., Teodorescu HN., Fukuda T, (Eds.), Systematic Organisation of Information in Fuzzy Systems, NATO Sci. Series III, vol. 184, 2003, pp. 7-10.

28. TEODORESCU H. N., Parallelizing Dynamic Models Based on Fuzzy Coupled Map Networks. Proc. 4th IEEE Int. W. Intell. Data Acquisition \& Adv. Comp. Systems, Dortmund, Sept. 06-08, 2007, pp. 170-175.

29. TEODORESCU H. N., On Fuzzy Sequences, Fixed Points and Periodicity in Iterated Fuzzy Maps. Int. J. Comp., Comm. \& Control, Vol. 6, 4, 2011, pp. 748-759.

30. TEODORESCU H. N., A. KANDEL, M. SCHNEIDER, Fuzzy Modeling and Dynamics. Fuzzy Sets and Systems, Vol. 106, 1, Aug. 16, 1999, pp. 1-2.

31. TEODORESCU H. N., A. KANDEL, L. HALL, Report of Research Activities in Fuzzy AI and Medicine at USFCSE. AI in Medicine, Vol. 21, 1-3, 2001, pp. 177-183.

32. TEODORESCU H. M., M. D. ZBANCIOC, L. PISTOL, Parallelizing Neuro-fuzzy Economic Models in a GRID Environment, Studies in Informatics and Control, Vol. 17, 1, 2008.

33. ZADEH, L. A., Fuzzy Logic = Computing with Words. IEEE Trans. Fuzzy Systems, Vol. 4, 2, 1996, pp. 103-111. 